\title{
Path probability for a Brownian motion
}

\author{
LIN TongLing $^{1,2}$, PUJOS Cyril ${ }^{1}$, OU CongJie ${ }^{3}$, BI WenPing $^{4}$, CALVAYRAC Florent $^{2} \&$ \\ WANG Qiuping Alexandre ${ }^{1,2^{*}}$ \\ ${ }^{1}$ LUNAM Université, ISMANS, Laboratoire de Physique Statistique et Systèmes Complexes, 44, Avenue F.A. Bartholdi, 72000 Le Mans, France; \\ ${ }^{2}$ LPEC, Université du Maine, Ave. O. Messiaen, 72035 Le Mans, France; \\ ${ }^{3}$ College of Information Science and Engineering, Huaqiao University, Quanzhou 362021, China; \\ ${ }^{4}$ Laboratoire d'Acoustique, Université du Maine, Ave. O. Messiaen, 72085 Le Mans Cedex 9, France
}

Received May 18, 2011; accepted July 19, 2011

\begin{abstract}
This work reports on numerical simulations of Brownian motion in the non-dissipative limit. The objective was to prove the existence of path probability and to compute probability values for some sample paths. By simulating a large number of particles moving from point to point under Gaussian noise and conservative forces, we numerically determine that the path probability decreases exponentially with increasing Lagrangian action of the paths.
\end{abstract}

classical Brownian motion, path probability, Lagrangian action, Hamiltonian action

Citation: $\quad$ Lin T L, Pujos C, Ou C J, et al. Path probability for a Brownian motion. Chinese Sci Bull, 2011, 56: 3736-3740, doi: 10.1007/s11434-011-4803-6

Brownian motion is an idealized approximation to actual random dynamics that has been extensively investigated over a long period time [1-3] but possibly still not thoroughly understood. Recently, Brownian and random motion have been extended into the field of fractional Brownian motion [4,5], stochastic noise [6,7], and quantum random walks $[8,9]$. One of the least studied aspects is the probability distribution of paths or trajectories. To be more precise, a Brownian particle can go from one configuration point to another along different trajectories, an important characteristic of random dynamics [10]. With that viewpoint, several questions arise. First, does a probability distribution for each configuration path exist? i.e., can a Brownian particle repeat the same path (or a bundle of paths with nonzero thickness)? Second, if a probability exists, what are the mechanical variables of the distribution? In other words, which quantities determine the frequency of repetition for the motion along the same bundle of paths?

The objective of the present work is to investigate by numerical simulation the path probability of random motion in the non or weak dissipative limit to find answers to both

*Corresponding author (email: awang@ismans.fr) questions. The method is simple. In answering the first question, one should choose several bundles of paths and observe whether Brownian particles retrace any of these. If paths are retraced, one must count the number of particles traversing each bundle of paths, and calculate mechanical quantities such as velocity, kinetic and potential energy, actions or some function of these quantities to find possible correlations between them and the path count.

A crucial issue about these quantities for Brownian motion is whether the time derivative of position exists, or whether the trajectories are time differentiable. According to a widely accepted point of view [11,12], trajectories corresponding to Brownian motion are not differentiable. Hence no velocity can exist. However, according to the Ornstein-Uhlenbeck model [13], the configuration trajectories are differentiable so an instantaneous velocity exists. It is worth mentioning a recent experiment [14] that recorded the one-dimensional individual trajectories of a 3- $\mu \mathrm{m}$ diameter silicon bead undergoing Brownian motion in air under low pressures and ambient temperatures. The conclusion of the work is that, if the time step of the position measurement is much smaller than the relaxation time of the motion, the trajectories corresponding to Brownian motion loose 
progressively their diffusive (self-similar) property and become smooth, ballistic, and differentiable, for which the definition $v=\Delta x / \Delta t$ does make sense if $\Delta t$ is sufficiently small.

To avoid these conceptual issues based on the mathematical models of motion, we suppose here that the considered motion is suitably smooth and that the velocity defined with coarse graining in configuration space with sufficiently small time scale can be a good approximation of the instantaneous motion. Hence we can define an instantaneous kinetic energy $K$, a potential energy $V$, the Lagrangian $L=$ $K-V$, Hamiltonian $H=K+V$, as well as the following two associated actions for any considered path $[a, b]$, $A_{\mathrm{L}}=\int_{a}^{b}(K-V) \mathrm{d} t$ and $A_{\mathrm{H}}=\int_{a}^{b}(K+V) \mathrm{d} t$. With this background, we describe below first the technical details of the numerical computation and second we present the numerically observed probability distributions of paths for a free particle, and particles subject to different forces. The main result is that the path probability decreases exponentially with increasing Lagrangian action but has no correlation with the Hamiltonian action.

\section{Numerical simulation}

We consider a large number $\left(\sim 10^{9}\right)$ of particles of mass $m$ undertaking one-dimensional stochastic motion in a conservative force field. From an initial point $x_{a}$, the particles can go anywhere along the $x$-axis. We are interested in the $N$ particles that arrive at a given point $x_{b}$ after $n$ steps. Because the motion is stochastic, these can take different paths from $x_{a}$ to $x_{b}$. A path is defined by a sequence of random positions $\left\{x_{a}, x_{1}, x_{2} \cdots x_{n-1}, x_{b}\right\}$, where $x_{i}$ is the position at time $t_{i}$ with $x_{a}=x_{0}$ and $x_{b}=x_{n}$. We chose $n=10$ in the present work with an equal unit time increment at each step, i.e. $\Delta t_{i}=t_{i}-t_{i-1}=1$ for every step $i$.

Our model of the motion is constructed based on the free particle solution of the diffusion equation of Fokker-Planck given by

$$
p\left(x_{i}-x_{i-1}, t_{i}-t_{i-1}\right) \propto \mathrm{e}^{-\frac{\left(x_{i}-x_{i-1}\right)^{2}}{2 D\left(t_{i}-t_{i-1}\right)}},
$$

Hence, for free particles, the sequence $\left\{x_{a}, x_{1}, x_{2} \cdots x_{n-1}, x_{b}\right\}$ can be generated by a Gaussian noise in the following way:

$$
x_{i+1}=x_{i}+\lambda \chi_{i}
$$

where $\lambda$ is a parameter adjusting the magnitude of the random displacement $x_{i+1}-x_{i}, \chi_{i}$ is a Gaussian-distributed noise generated by the computer at each step $i$.

For particles in conservative force fields, there is no general solution of the diffusion equation, and the forced motion should be introduced directly into the displacements of each step. Our model consists in separating the motion into two parts: a random part given by eq. (2) and a part described by a motion equation $y_{i}=f\left(t_{i}\right)$ derived from the Newtonian equation of motion under a conservative force. The total displacement of each step is then given by

$$
x_{i+1}=x_{i}+\lambda \chi_{i}+f\left(t_{i}\right) \text {. }
$$

Figure 1 illustrates some sample paths generated by eq. (3) with constant force (for example weight). These samples are chosen among a large number of paths in such a way that these include the least action path and those paths around the least action for which the values actions are sufficiently close but no too much different from one. Each sample path is given a small thickness $\delta$ (for example $\delta=0.06$ for a total variation in $x$ of about 1 or 2 ) to form a bundle or a tube of paths. From here on, we will call this bundle a path. Obviously, the larger $\delta$ is for a path, the more particles there are moving along the path from $a$ to $b$.

The numerical simulation mainly consists in observing the total number of particles $N$ moving from $a$ to $b$ through whichever path and the number of particles $N_{k}$ moving along a given sample path $k$ from $a$ to $b$. The probability that the path $k$ is taken is determined by $P_{k}=N_{k} / N$ (with large $N$ ).

For each sample path, we calculate the Lagrangian and Hamiltonian actions. For this purpose, the velocity at time step $i$ is defined as $v_{i}=\frac{x_{i}-x_{i-1}}{t_{i}-t_{i-1}}$. The kinetic energy is then given by $K_{i}=\frac{1}{2} m v_{i}^{2}$, and the two actions by $A_{\mathrm{L}}=\sum_{i=1}^{10}\left[\frac{1}{2} m v_{i}^{2}-V\left(x_{i}\right)\right] \cdot \Delta t_{i} \quad$ and $\quad A_{\mathrm{H}}=\sum_{i=1}^{10}\left[\frac{1}{2} m v_{i}^{2}+V\left(x_{i}\right)\right] \cdot \Delta t_{i}$.

The magnitudes of the displacements and forces are chosen

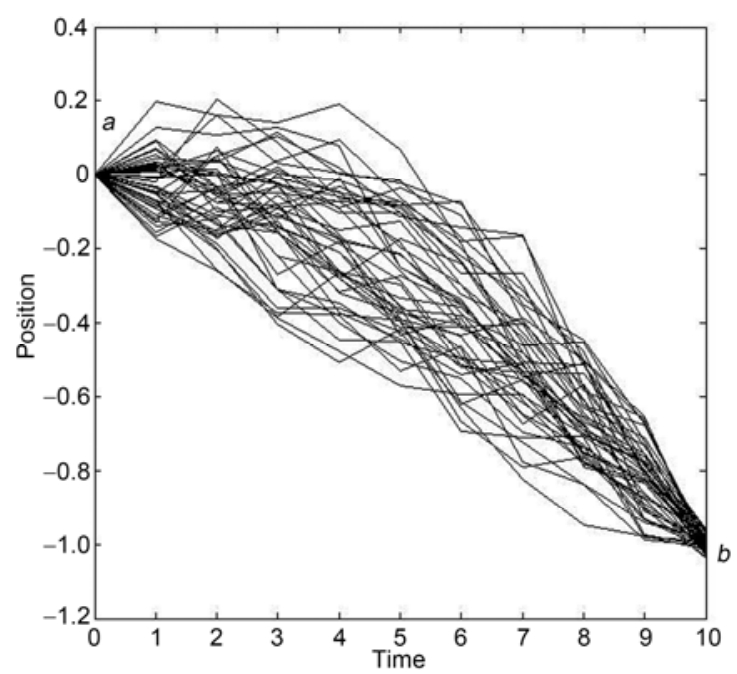

Figure 1 Example of paths (bundle) from point $a$ to point $b$ for particles in a constant force field such as weight. The time duration of the motion is $n=10$ steps with the same unit time increment for each step. Each path is a sequence of positions $\left\{x_{a}, x_{1}, x_{2} \cdots x_{n-1}, x_{b}\right\}$. 
such that the kinetic and potential energies are of the same order of magnitude to distinguish the maximum of the two actions along the same path.

Simulations were performed with several one-dimensional potential energies. We considered free particles with $V(x)=0$, particles under a constant force with $V(x)=m g x$, under a harmonic force with $V(x)=\frac{1}{2} m w^{2} x^{2}$, and other more complicated force potentials with $V(x)=\frac{2}{\sigma} x^{3}$ and $V(x)=-\frac{1}{2} \sigma^{2}(1-x)^{4} \quad(\sigma>0)$ to check the generality of the results. The results of the simulations are presented below.

\section{Determination of path probability distributions}

To find any correlation between the path probability and the Lagrangian and Hamiltonian actions, the probability is plotted against both. In each figure, the action is place along the abscissa for ease in comparing the probability dependence. We did not introduce the time scale or the diffusion constant of real Brownian motion, because the value of the time increment of each step does not have any physical sense and is taken as unity. We tried values different from unity but that of course did not alter the results. In this case, the scale of the displacements is also arbitrary and the actions do not have unity. The important aspect here is to find, under the assumption of the existence of instantaneous velocity and kinetic energy, the dependence of the path probability on the Lagrangian or Hamiltonian action. The exact numerical relationship is not of interest in this work.

\subsection{Free particles}

For free particles, the potential energy is zero; there is no difference between the Lagrangian and Hamiltonian actions. From Figure 2, it is obvious that the probability for a path $k$ to occur is given by

$$
p_{k}(A)=\frac{1}{Z} \mathrm{e}^{-\gamma A_{k}}
$$

where $A_{k}$ is either the Lagrangian or Hamiltonian action of a path $k$ between the $a$ and $b$. The slope is found to be around $\gamma=80$. The normalization function $Z$ in eq. (4) can be determined analytically with the normalization by the path integral [13]

$$
\int_{-\infty}^{\infty} \frac{\mathrm{d} x_{1}}{\delta} \int_{-\infty}^{\infty} \frac{\mathrm{d} x_{2}}{\delta} \int_{-\infty}^{\infty} \frac{\mathrm{d} x_{3}}{\delta} \ldots \int_{-\infty}^{\infty} \frac{\mathrm{d} x_{n-1}}{\delta} p_{k}\left(A_{k}\right)=1
$$

with fixed $x_{a}$ and $x_{b}$, or numerically by the value of $\ln p(A=0)$ which can be found with the distribution curves in the following figures.

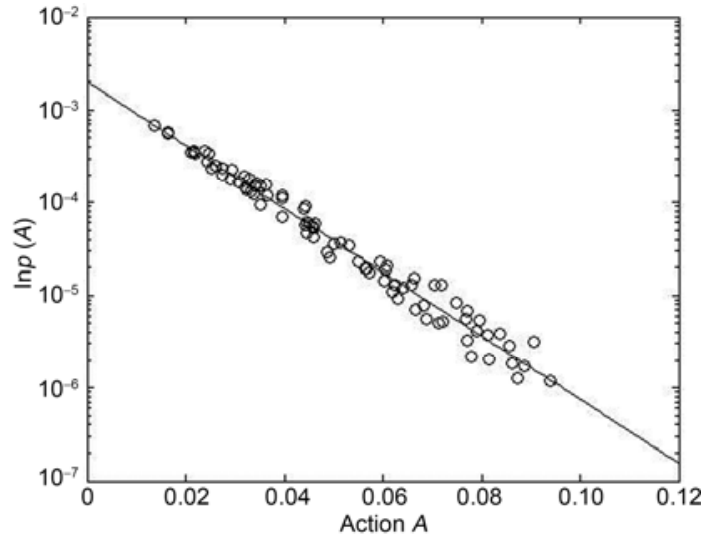

Figure 2 Path probability distribution against the Lagrangian (equivalently Hamiltonian) action for a free particle with $V(x)=0$. The straight line is a best fit of the points; the slope is about $\gamma=80$.

\subsection{Particles under constant force}

To understand the dependence of the path probability on Lagrangian and Hamiltonian actions, it is necessary to study Brownian particles under the action of conservative forces. The first force we studied is the constant force obtained from potential $V(x)=g x$, and giving displacement $x(t)=-\frac{1}{2} g t^{2}$. The value of parameter $g$ is chosen to be $g=0.02$ so that, within 10 time steps, the final point $b$ is not too far from $a$. The results are shown in Figure 3. Eq.(4) still holds with the slope $\gamma=71$. It is obvious from the figure that with increasing Lagrangian action the path probability decreases exponentially. However, there is no apparent correlation between path probability and Hamiltonian action. The least value for the Lagrangian action is around 0.14 .

\subsection{Particles under harmonic force}

The potential of the harmonic force is $V(x)=\frac{1}{2} \omega^{2} x^{2}$ giving



Figure 3 Path probability distribution against the Lagrangian (circles) and Hamiltonian (stars) actions for particles under constant force with potential $V(x)=m g x$. The path probability decreases exponentially with increasing Lagrangian action. There is no correlation between the path probability and Hamiltonian action. 
$x(t)=A \sin (\omega t), A=1$ and $\omega=3 \pi / 2 n$. Figure 4 shows the path probability distribution against both actions which for the Lagrangian action yields a slope of $\gamma=113$. As for constant force, the path probability distribution decreases exponentially with increasing Lagrangian action with no correlation with the Hamiltonian action.

\subsection{Particles in other conservative potentials}

One might ask from the results presented above whether under the two special forces (weight and elastic) the straight lines are exceptions. Hence, it would be interesting to demonstrate that the exponential of Lagrangian action yields a universal path probability at least in these numerical experiments using Gaussian noise. Lacking general mathematical proof, we were content in simulations to use higher-order polynomial potential functions such as $V(x)=\frac{2}{\sigma} x^{3} \quad$ where $\quad x(t)=-\frac{\sigma}{(1+t)^{2}} \quad(\sigma=1.2 \quad) \quad$ and $V(x)=-\frac{1}{2} \sigma^{2}(1-x)^{4} \quad$ where $x(t)=\frac{\sigma t}{1+\sigma t} \quad(\sigma=1)$. The path probability distributions against the two actions are shown in the Figures 5 and 6. From these figures, eq. (4) always holds for the Lagrangian action with exponent with $\gamma=24$ for the cubic potential, and $\gamma=59$ for the quartic.

\section{Discussion}

Looking at the above figures, we can suggest an exponential correlation of the Lagrangian action with the path probability. A more convincing argument can be given by considering the following correlation function between the actions $A\left(A_{\mathrm{L}}\right.$ or $\left.A_{\mathrm{H}}\right)$ and $-\ln P(A)$ given by



Figure 4 Path probability distribution against Lagrangian (circles) and Hamiltonian action (stars) for particles in a harmonic potential $V(x)$ $=\frac{1}{2} \omega^{2} x^{2}$.

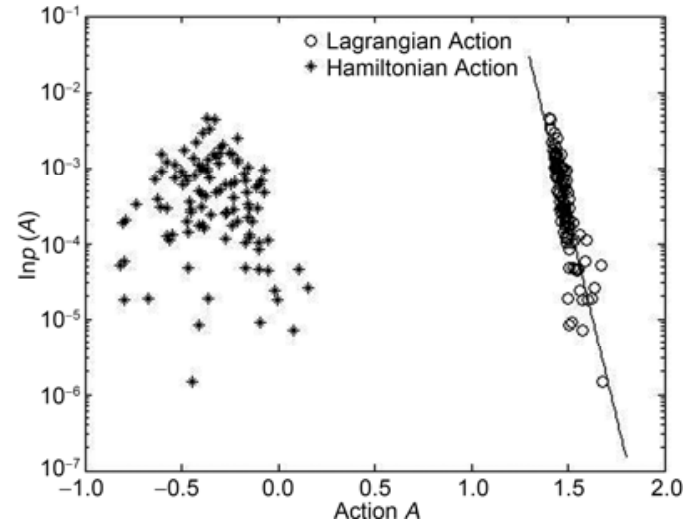

Figure 5 Path probability distribution against Lagrangian (circles) and Hamiltonian actions (stars) for particles in potential $V(x)=\frac{2}{\sigma} x^{3}$.

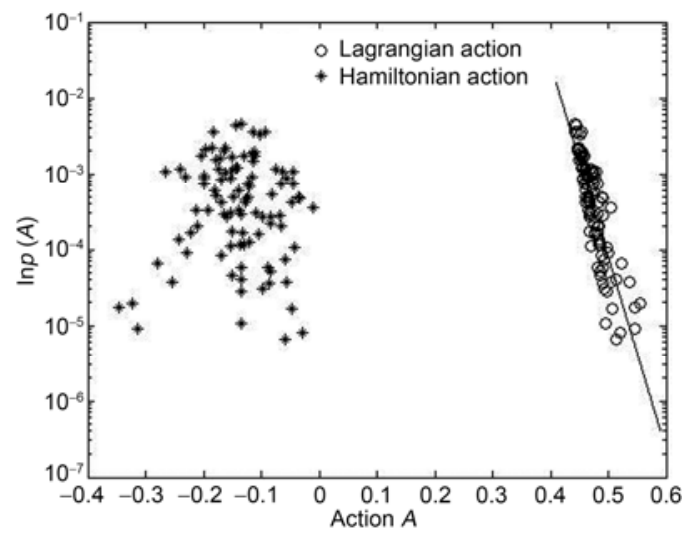

Figure 6 Path probability distribution against Lagrangian (circles) and Hamiltonian action (stars) for particles in potential $V(x)=-\frac{1}{2} \sigma^{2}(1-x)^{4}$.

$$
c(A)=\frac{\sum_{i=1}^{n}\left(A_{i}-<A>\right)\left(-\ln P\left(A_{i}\right)+<\ln P\left(A_{i}\right)>\right)}{\sqrt{\left[\sum_{i=1}^{n}\left(A_{i}-<A>\right)^{2}\right]\left[\sum_{i=1}^{n}\left(-\ln P\left(A_{i}\right)+<\ln P\left(A_{i}\right)>\right)^{2}\right]}},
$$

where $\langle A\rangle$ is the mean of action $A$, and $\left\langle\ln P\left(A_{i}\right)>\right.$ the mean of $-\ln P(A) . c(A,-\ln P(A)) \approx 1$ (or $c(A,-\ln P(A)) \approx 0$ ) would indicate that $A$ and $-\ln P(A)$ are linearly correlated (uncorrelated); the calculation results are shown in Table 1.

Table 1 Correlation function $c(A)$ for different potentials

\begin{tabular}{lcc}
\hline Potentials & \multicolumn{2}{c}{ Correlation } \\
\cline { 2 - 3 } & $c\left(A_{\mathrm{L}}\right)$ & $c\left(A_{\mathrm{H}}\right)$ \\
$V(x)=0$ & 0.9928 & 0.9928 \\
$V(x)=g x$ & 0.9487 & 0.4137 \\
$V(x)=\frac{1}{2} \omega^{2} x^{2}$ & 0.9907 & 0.2161 \\
$V(x)=\frac{2}{\sigma} x^{3}$ & 0.8466 & 0.0118 \\
$V(x)=-\frac{1}{2} \sigma^{2}(1-x)^{4}$ & 0.8629 & 0.0415 \\
\hline
\end{tabular}


It can be concluded from Table 1 that $-\ln P(A)$ is more strongly correlated linearly with the Lagrangian action than the Hamiltonian action.

The result would be more precise and convincing if we could use more particles and longer simulation times. However, it is difficult because of limited computer speeds. Another possible improvement is to simulate real Brownian motion using particles in a gas or liquid instead of using Gaussian noise. It is also an appealing idea to investigate the path probability distribution when the position distribution is not Gaussian. This work is in progress.

\section{Conclusion}

We numerically simulated Brownian motion in the limit of non-dissipative case. We observed a large number of particles (up to $10^{9}$ ) moving from one point to another along different paths within a given number of time steps (up to 10) and computed for each path (bundle) the path probability distribution using the Lagrangian and Hamiltonian actions. We found that the path probability depends exponentially on the Lagrangian action rather than the Hamiltonian action as believed by some. The least action path is just the most probable one.

Finally, we would like to stress that, although in this work we did not use the space-time scales of true Brownian diffusion, the exponential dependence of the path probability based on the Lagrangian action seems not to be accidental. Added support is given by a theoretical prediction obtained by a stochastic least action principle in our previous work [15-18]. It is also possible to develop a theoretical framework for random mechanical motion on the basis of this action principle [19]. Within this framework, an interpretation of the second law of thermodynamics (time arrow) has been formulated on the basis of a modified Liouville theorem concerning the conservation of the phase space distribution $[10,19]$.

This work was supported by the Region des Pays de la Loire in France
(2007-6088 and 2009-09333).

1 Einstein A. On the movement of small particles suspended in a stationary liquid demanded by the molecular kinetic theory of heat. Ann Phys, 1905, 17: 549-560

2 Einstein A. On the theory of the Brownian movement. Ann Phys, 1906, 19: 371-381

3 Duplantier B. Le mouvement brownien, 'divers et ondoyant'. Séminaire Poincaré, 2005, 1: 155-212

4 Luan N N. Hausdorff measures of the image, graph and level set of bifractional Brownian motion. Sci China Math, 2010, 53: 2973-2992

5 Liu G Y, Zhang X S. Asymptotic properties of power variations for fractional Brownian motion with jumps. Sci China Math, 2011, 41: 81-94

6 Liu X P, Liu K H. The generalization of a class of impulse stochastic control models of a geometric Brownian motion. Sci China Ser F-Inf Sci, 2009, 52: 983-998

7 Peng S G. Survey on normal distributions, central limit theorem, Brownian motion, and the related stochastic calculus under sublinear expectations. Sci China Ser A-Math, 2009, 52: 1391-1411

8 Wang C, Li Y S, Hao L. Optical implementation of quantum random walks using weak cross-Kerr media. Chinese Sci Bull, 2011, 56: 2088-2091

9 Liu H, Liu J S, Lü J T, et al. Polarization-dependent difference of the power spectra from two-dimensional random media with different shapes. Chinese Sci Bull, 2009, 54: 3215-3219

10 Wang Q A. What can we still learn from Brownian motion? In: Proceedings of the First Franco-Mongolian Workshop on Material Sciences, August 22-25, 2010. Ulaabaatar, Mongolia: Tsomirlog Press, 2010. 80

11 Nelson E. Derivation of the Schrödinger equation from Newtonian mechanics. Phys Rev, 1966, 150: 1079-1085

12 Feynman R P, Hibbs A R. Quantum Mechanics and Path Integrals. New York: McGraw-Hill Publishing Company, 1965

13 Uhlenbeck G E, Ornstein L S. On the theory of Brownian motion. Phys Rev, 1930, 36: 823-841

14 Li T C, Kheifets S, Medellin D, et al. Measurement of the instantaneous velocity of a Brownian particle. Science, 2010, 328: 16731675

15 Wang Q A. Maximum path information and the principle of least action for chaotic system. Chaos Solit Fract, 2004, 23: 1253-1258

16 Wang Q A. Non quantum uncertainty relations of stochastic dynamics. Chaos Solit Fract, 2005, 26: 1045-1052

17 Wang Q A. Maximum entropy change and least action principle for nonequilibrium systems. Astrophys Space Sci, 2006, 305: 273-281

18 Wang Q A, Tsobnang F, Bangoup S, et al. Reformulation of a stochastic action principle for irregular dynamics. Chaos Solit Fract, 2009, 40: 2550-2556

19 Wang Q A. A probabilistic mechanics theory for random dynamics. arXiv: 1002.4026

Open Access This article is distributed under the terms of the Creative Commons Attribution License which permits any use, distribution, and reproduction in any medium, provided the original author(s) and source are credited. 